\title{
Evolution spatiale de l'intelligibilité de la parole dans un habitacle automobile
}

\author{
E. PARIZET
}

Direction de la Recherche de Renault, 9-11 avenue du 18 Juin 1940, 92500 Rueil-Malmaison, France

\begin{abstract}
In a car, speech intelligibility primarily depends on the ratio between speech and noise levels. The speech level, as perceived by the listener, is influenced by the compared positions of the speaker and the listener and the characteristics of the car cabin (its geometry and materials).

In this study, the Articulation Index was used to evaluate speech intelligibility in different positions in the compartment of a luxury sedan car, for two sound sources : an omnidirectionnal one and a loudspeaker whose directivity is the same as a human speaker's.

The effect of human's directivity is to decrease speech intelligibility, especially in cases where the communication is difficult (from the front seats to the rear ones).

These measurements were also compared with simple models of sound field in the car (free field and diffuse field). For an omnidirectional source, the field is nearly a free one; for a directional one, the surfaces of the cabin can reflect sounds to increase the level, but this increase is not equivalent to what can be seen in a diffuse field.

Therefore, the geometry and absorption characteristics in a car compartment could be modified in order to improve communication between passengers.
\end{abstract}

\section{INTRODUCTION}

La facilité avec laquelle les passagers d'un véhicule peuvent converser est pour eux un élément important de confort. Cette conversation n'est pas toujours aisée, surtout lorsque les passagers assis à 1'arrière doivent comprendre ceux assis à l'avant.

Le paramètre essentiel est ici le rapport d'énergie entre la voix du locuteur et le bruit perturbateur, créé par le véhicule. Les progrès des constructeurs automobiles font que le niveau de ce bruit est en perpétuelle décroissance; cependant, comme les habitacles sont de plus en plus spacieux, bien séparés en deux compartiments par les sièges avant et abondamment garnis de matériaux très absorbants, le niveau de la parole perçue par l'auditeur diminue également.

L'objet de ce travail est d'évaluer la variation d'intelligibilité dans un habitacle automobile et d'apprécier l'influence de cet habitacle sur la propagation de la voix du locuteur aux auditeurs. 


\section{METHODE}

\subsection{Présentation de l'habitacle}

Un habitacle automobile est un local de très petites dimensions (volume compris entre 2 et $3 \mathrm{~m}^{3}$ ). Les parois de ce local, dont l'aire totale est voisine de $20 \mathrm{~m}^{2}$, peuvent être très réfléchissantes (c'est le cas des vitrages) ou très absorbantes, du moins pour les fréquences supérieures à $1 \mathrm{kHz}$, comme les sièges et la garniture de pavillon.

Dans l'exemple d'une Renault 25, les coefficients d'absorption des matériaux de l'habitacle ont été mesurés dans une petite chambre réverbérante; les résultats obtenus conduisent à des valeurs de la surface d'absorption totale comprises entre $3,2 \mathrm{~m}^{2}$ à $125 \mathrm{~Hz}$ et $10 \mathrm{~m}^{2}$ à $4 \mathrm{kHz}$.

\subsection{Estimation de l'intelligibilité}

Nous avons utilisé l'indicateur le plus couramment utilisé, qui est l'Indice d'Articulation mis au point par French et Steinberg (1). C'est une moyenne pondérée des différences d'énergie entre parole et bruit, dans les bandes de fréquences comprises entre 160 et $8 \mathrm{kHz}$. De nombreuses pondérations ont été proposées pour décrire au mieux l'importance des différentes fréquences de la voix dans sa compréhension; cependant, dans le cas particulier des bruits intérieurs d'automobiles, elles conduisent toutes à des résultats équivalents (2).

Il existe certes des indicateurs plus sophistiqués, comme 1'Indice de Transmission de la Parole ou STI (3), qui tient également compte de la déformation du message par le local. Cependant, les réponses impulsionnelles mesurées dans un habitacle automobile sont tellement brèves que le STI prend des valeurs maximales dans une voiture à l'arrêt; cet indice est donc comparable à l'Indice d'Articulation.

\subsection{Cartographies de l'Indice d'Articulation}

Dans une Renault 25 à l'arrêt, nous avons placé sous le pavillon un cadre rectangulaire, long de 1,65 m et large de 1,2 m. Ce cadre entoure la zone dans laquelle se trouvent les passagers (il va du sommet du pare-brise à celui de la glace arrière et d'un côté à l'autre de l'habitacle). Une tige déplaçable permet alors d'installer un microphone sur tous les points d'un quadrillage intérieur au cadre et de pas $15 \mathrm{~cm}$ (sauf sous les coins du cadre, ce qui donne un total de 84 points de mesure).

Une source sonore peut alors être placée à la position d'un locuteur; elle émet du bruit rose à un niveau calibré. En chaque position du microphone, le spectre tiers d'octave du bruit capté par le microphone est calculé par un ordinateur PC supportant une carte d'acquisition, ainsi que la différence entre l'Indice d'Articulation obtenu en ce point et celui mesuré en une position de référence, choisie à $50 \mathrm{~cm}$ en face de la source (dans la direction d'émissivité maximale de celle-ci). On obtient ainsi des valeurs qui ne sont plus comprises entre 0 et 1 comme les valeurs usuelles de 1'Indice d'Articulation; ces valeurs sont enfin représentées sur le plan de mesure, après un lissage spatial.

Cette méthode suppose que le niveau de bruit est uniforme dans l'habitacle, ce qui n'est pas rigoureusement exact; mais elle a l'avantage de la simplicité (puisqu'on s'affranchit des caractéristiques fréquentielles du signal émis par la source) et permet d'apprécier l'effet propre de l'habitacle sur l'intelligibilité.

\section{RESULTATS}

\subsection{Source omnidirectionnelle}

La figure 1 présente les résultats obtenus pour une source omnidirectionnelle placée à l'avant gauche puis à l'arrière gauche (pour toutes les cartes présentées, l'habitacle est vu de dessus, l'avant du véhicule est donc en haut de la figure). On constate que les deux cartes de la figure 1 sont à peu près symétriques selon l'axe 
transversal du véhicule. Les pertes d'intelligibilité sont très faibles pour le passager assis à côté du locuteur et valent $-0,2$ à $-0,3$ pour les autres passagers.
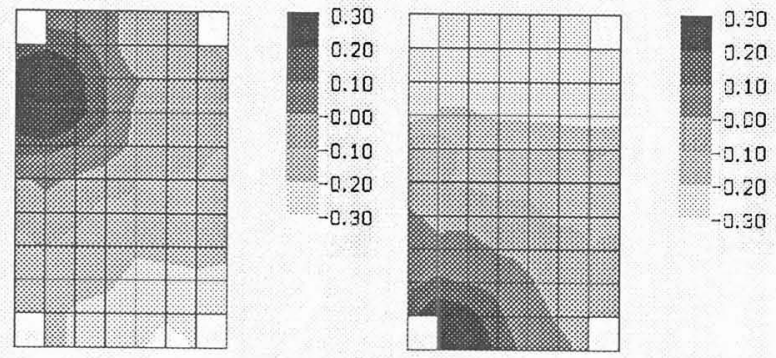

Figure 1 : Indices d'Articulation dans le véhicule, pour une source omnidirectionnelle à l'avant gauche et à l'arrière gauche

\subsection{Source directionnelle}

Un locuteur humain est une source très directionnelle, surtout aux hautes fréquences (4) et (5). L'atténuation maximale, obtenue dans le plan horizontal pour un azimut de $150^{\circ}$, varie entre $6 \mathrm{~dB}$ (à $125 \mathrm{~Hz}$ ) et $15 \mathrm{~dB}($ à $4 \mathrm{kHz})$.

Pour reproduire cette directivité, nous avons utilisé une enceinte sphérique de diamètre environ $30 \mathrm{~cm}$. Lorsque cette source est placée en position de conducteur (son axe d'émissivité maximale étant dirigé bien sûr vers l'avant), on constate (figure 2) une perte d'intelligibilité nette pour les passagers avant droit $(-0,3)$ et surtout arrière $(-0.5$ à -0.6$)$.

Lorsqu'elle est placée en position de passager arrière gauche, la communication est meilleure dans tout l'habitacle, puisque la source est cette fois dans une orientation favorable.
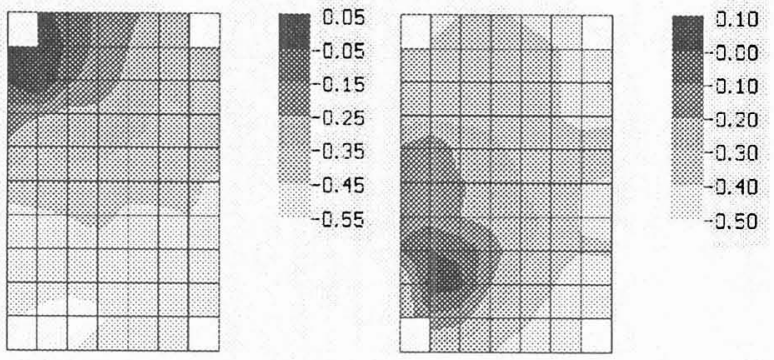

Figure 2 :Indices d'Articulation dans le véhicule, pour une source directionnelle à l'avant gauche et à l'arrière gauche

\section{COMPARAISONS AVEC DES MODELES SIMPLES DE CHAMP SONORE}

L'énergie captée dans un local, en un point à une distance $r$ et sous une incidence $\theta$ d'une source rayonnant une puissance $W$ avec un facteur de directivité $Q_{\theta}$ vaut $p_{\text {mis }}^{2}(r, \theta)=\rho c W\left[\frac{Q_{\theta}}{4 \pi r^{2}}+\frac{4}{A}\right]$, où $c$ est la célérité du son, $\rho$ la densité de l'air et A la surface d'absorption totale équivalente (6). Le premier terme entre crochets représente le champ direct rayonné par la source et le second la partie réverbérée par la salle.

Cette expression permet de calculer la différence d'énergie entre tout point de l'habitacle et le point de référence (pour lequel $\mathrm{r}=0,5 \mathrm{~m}$ et $\theta=0$ ) dans chaque bande de fréquence, ce qui conduit à la différence d'Indice d'Articulation. 
Pour une source omnidirectionnelle, $\mathrm{Q}_{\theta}$ vaut constamment 1 . La figure 3 montre la comparaison entre les résultats obtenus lorsque cette source est placée dans la voiture en position de conducteur (à gauche) et ceux calculés en simulant un champ libre dans l'habitacle (au centre) et un champ diffus (à droite). Cette dernière carte a été obtenue en utilisant les valeurs expérimentales des coefficients d'absorption des divers matériaux de l'habitacle. On constate que le champ expérimental est très proche d'un champ libre, surtout pour les passagers arrière.
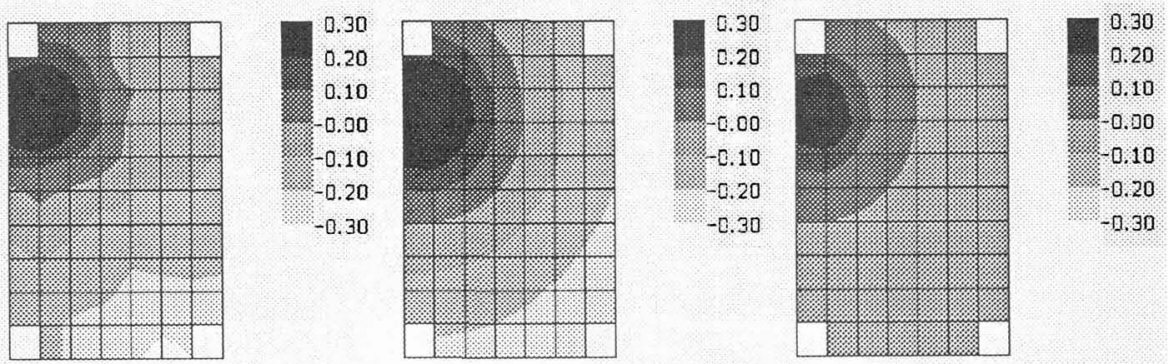

Figure 3 : Indices d'Articulation obtenus pour une source omnidirectionnelle, dans le véhicule, en champ libre et en champ diffus

Pour un locuteur humain (figure 4), l'habitacle renforce l'énergie de la parole reçue à l'arrière du véhicule; cet accroissement est cependant encore loin de ce qui aurait été obtenu en champ diffus.
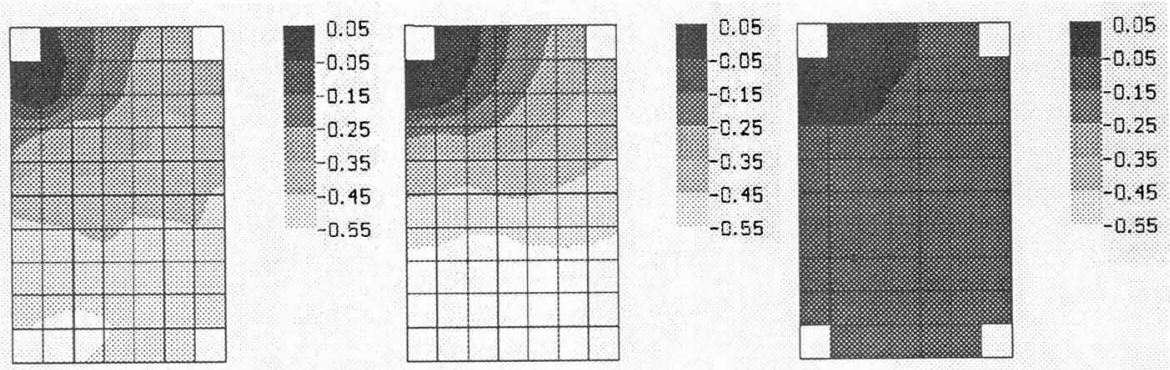

Figure 4 : Indices d'Articulation obtenus pour une source directionnelle, dans le véhicule, en champ libre et en champ diffus

\section{CONCLUSION}

Alors que le champ créé par le bruit de fonctionnement du véhicule est proche d'un champ diffus (7), celui obtenu lorsqu'un passager parle s'apparente plus à un champ libre. Cependant, l'habitacle peut favoriser la communication, surtout lorsqu'on respecte la directionnalité de la source, qui est un facteur d'affaiblissement de l'intelligibilité.

Il y a donc un enjeu à optimiser l'habitacle automobile, en jouant sur sa géométrie intérieure et surtout sur les caractéristiques d'absorption des matériaux, pour améliorer la communication entre les passagers.

\section{References}

[1] French N.R. et Steinberg J.C., J. Acoust. Soc. Am. 19 (1947) 90-119.

[2] Parizet E., Noise Con. Eng. J. (1992) 38 73-79.

[3] Houtgast T. et Steeneken H.J.M, Acustica 28 (1984) 66-73.

[4] Moreno A. et Pfretszshner J., J. Elec. Fis. Apl. 14 (1971) 369-371.

[5] McKendree F.S., Proc. InterNoise 86 (1986) 911-915.

[6] Beranek L.L., Noise and Vibration Control (McGraw Hill Company, New York, 1971) pp. 224-230.

[7] Dal Degan N. et Prati C., Signal Processing 15 (1988) 43-56. 